\title{
Certain geometric properties of Mittag-Leffler functions
}

\author{
Saddaf Noreen ${ }^{1}$, Mohsan Raza ${ }^{1 *}$ (1) and Sarfraz Nawaz Malik²
}

\section{"Correspondence:}

mohsan976@yahoo.com

'Department of Mathematics, Government College University

Faisalabad, Faisalabad, Pakistan Full list of author information is

available at the end of the article

\section{Springer}

\begin{abstract}
In this paper, some geometric properties of normalized Mittag-Leffler functions are investigated. We focus on starlikeness of order $2 \mu+\eta-1$ and convexity in the direction of imaginary axis. In addition, we study pre-starlikeness of Mittag-Leffler functions. The results are obtained by using the positivity technique.

MSC: 33E12; 30C45

Keywords: Mittag-Leffler functions; Starlike functions; Close-to-convex functions; Typically real function; Convex functions in the direction of imaginary axis; Pre-starlike functions
\end{abstract}

\section{Introduction}

Recently, there has been overwhelming interest in the study of Mittag-Leffler functions. The Mittag-Leffler type functions have widespread applications in physics, biology, chemistry, engineering, and some other applied sciences. Some other aspects of the applications of these functions can be seen in fractional differential equations, stochastic systems, dynamical systems, statistical distributions, and chaotic systems. The geometric properties like starlikeness, convexity, and close-to-convexity of these functions have been investigated on a large scale by a number of researchers. We can easily see the direct usage of these functions to many techniques of fractional calculus. Gorenflo et al. [9], Kilbas et al. [14], and Srivastava et al. [34, 35] are a few leading precedents of these contributions. The Swedish mathematician G. M. Mittag-Leffler gave the idea of so-called Mittag-Leffler function $E_{\alpha}(z)$, see [17]. Later, it was studied by Wiman [37, 38] who defined it in terms of power series depending on the complex parameter $\alpha$. It may be regarded as a special function of $z \in \mathbb{C}$. We define it as follows:

$$
\mathbb{E}_{\alpha}(z)=\sum_{n=0}^{\infty} \frac{z^{n}}{\Gamma(\alpha n+1)}, \quad z \in \mathbb{C}, \alpha \in \mathbb{C} .
$$

We observe that the power series (1.1) converges in the whole complex plane for all $\operatorname{Re} \alpha>0$, whereas it diverges everywhere on $\mathbb{C} \backslash\{0\}$ for all $\operatorname{Re} \alpha<0$. We also note that for $\operatorname{Re} \alpha=0$, its radius of convergence is $R=e^{\pi / 2|\operatorname{Im}(z)|}$. Wiman [37, 38] gave the first twoparametric generalizations of the function defined in (1.1). It was later studied by Agarwal

(c) The Author(s) 2019. This article is distributed under the terms of the Creative Commons Attribution 4.0 International License (http://creativecommons.org/licenses/by/4.0/), which permits unrestricted use, distribution, and reproduction in any medium, provided you give appropriate credit to the original author(s) and the source, provide a link to the Creative Commons license, and indicate if changes were made. 
[4], Humbert [10], and Agarwal and Humbert [11]. We define it as follows:

$$
\mathbb{E}_{\alpha, \beta}(z)=\sum_{n=0}^{\infty} \frac{z^{n}}{\Gamma(\alpha n+\beta)}, \quad \alpha, \beta \in \mathbb{C}, \operatorname{Re}(\alpha)>0, \operatorname{Re}(\beta)>0, z \in \mathbb{C}
$$

For different values of parameters, the conditions of convergence vary. When $\alpha$ and $\beta$ are positive and real, the above given series converges in the entire complex plane. Another important and interesting fact about Mittag-Leffler functions is that the one and two-parametric Mittag-Leffler functions are fractional extensions of the basic functions. That is, $E_{1}( \pm z)=E_{1,1}( \pm z)=e^{ \pm z}, E_{1,2}(z)=\left(e^{z}-1\right) / z, E_{2}(z)=E_{2,1}(z)=\cosh (\sqrt{z}), E_{2,2}(z)=$ $\sinh (\sqrt{z}) / \sqrt{z}$. For more about Mittag-Leffler functions, see $[1,3,12]$.

Let $\mathcal{A}$ denote the class of functions $f$ of the form

$$
f(z)=z+\sum_{n=2}^{\infty} a_{n} z^{n}
$$

which are analytic in the open unit $\operatorname{disc} \mathcal{U}=\{z \in \mathbb{C}:|z|<1\}$. Let $\mathcal{S}$ denote the class of all functions in $\mathcal{A}$ which are univalent in $\mathcal{U}$. Let $\mathcal{S}^{*}(\mu), \mathcal{C}(\mu)$, and $\mathcal{K}(\mu)$ denote the classes of starlike, convex, and close-to-convex of order $\mu$, respectively, defined as follows:

$$
\begin{aligned}
& \mathcal{S}^{*}(\mu)=\left\{f: f \in \mathcal{A} \text { and } \operatorname{Re}\left(\frac{z f^{\prime}(z)}{f(z)}\right)>\mu, z \in \mathcal{U}, \mu \in[0,1)\right\} \\
& \mathcal{C}(\mu)=\left\{f: f \in \mathcal{A} \text { and } \operatorname{Re}\left(1+\frac{z f^{\prime \prime}(z)}{f^{\prime}(z)}\right)>\mu, z \in \mathcal{U}, \mu \in[0,1)\right\}
\end{aligned}
$$

and

$$
\mathcal{K}(\mu)=\left\{f: f \in \mathcal{A} \text { and } \operatorname{Re}\left(\frac{z f^{\prime}(z)}{g(z)}\right)>\mu, z \in \mathcal{U}, \mu \in[0,1), g \in \mathcal{S}^{*}\right\} .
$$

It is clear that

$$
\mathcal{S}^{*}(0)=\mathcal{S}^{*}, \quad \mathcal{C}(0)=\mathcal{C}, \quad \text { and } \quad \mathcal{K}(0)=\mathcal{K}
$$

The functions $z, \frac{z}{(1-z)}, \frac{z}{1-z^{2}}, \frac{z}{1+z}, \frac{z}{1+z^{2}}$ are starlike and univalent functions. It is convenient for $f$ to be close-to-convex, when the corresponding function $g$ has one of the aforementioned forms.

Consider a function $f \in \mathcal{A}$ which is real on the segment $(-1,1)$. If $f$ satisfies the relation

$$
\operatorname{Im}(z) \operatorname{Im} f(z)>0, \quad z \in \mathcal{U},
$$

then $f$ is called a typically real function. The class of typically real functions $\mathcal{T}$ was introduced by Robertson [26]. A function $f \in \mathcal{S}$ is said to be convex in the direction of imaginary axis if and only if the domain $f(\mathcal{U})$ is convex in the direction of imaginary axis. That is, for every $w_{1}, w_{2} \in f(\mathcal{U}),\left[w_{1}, w_{2}\right] \subset f(\mathcal{U})$ such that $\operatorname{Re} w_{1}=\operatorname{Re} w_{2}$. Robertson [26] showed that a function $f \in \mathcal{A}$ with real coefficients is convex in the direction of imaginary axis if $z f^{\prime}(z)$ is typically real. It is equivalent to

$$
\operatorname{Re}\left[\left(1-z^{2}\right) f^{\prime}(z)\right]>0, \quad z \in \mathcal{U} .
$$


Ruscheweyh [27] proved that if a function $f \in \mathcal{T}$ and satisfies $\operatorname{Re} f^{\prime}(z)>0$ for $z \in \mathcal{U}$, then $f$ is a starlike univalent function in $\mathcal{U}$. The extension of this definition up to order $\mu$ was given by Mondal and Swaminathan in [18].

Let $f \in \mathcal{A}$ of the form (1.3) and $g \in \mathcal{A}$ be given by

$$
g(z)=z+\sum_{n=2}^{\infty} b_{n} z^{n}
$$

Then convolution or Hadamard product of $f$ and $g$ is defined as

$$
(f * g)(z)=z+\sum_{n=2}^{\infty} a_{n} b_{n} z^{n} \quad(z \in \mathcal{U})
$$

We also focus on the class of pre-starlike functions, initiated in [28]. The class $\mathcal{R}_{\mu}$ denotes the class of pre-starlike functions of order $\mu$ and is defined as follows:

$$
\mathcal{R}_{\mu}=\left\{f: f \in \mathcal{A} \text { and }\left\{\begin{array}{l}
\operatorname{Re} \frac{f(z)}{z}>0, \quad z \in \mathcal{U} \text { for } \mu=1, \\
\frac{z}{(1-z)^{2(1-\mu)}} * f(z) \in \mathcal{S}^{*}(\mu), \quad z \in \mathcal{U} \text { for } 0 \leq \mu<1 .
\end{array}\right\}\right.
$$

In particular, $\mathcal{R}_{1 / 2}=\mathcal{S}^{*}(1 / 2)$ and $\mathcal{R}_{0}=\mathcal{C}$. Sheil-Small et al. [32] generalized the class $\mathcal{R}_{\mu}$ and defined the class $\mathcal{R}[\rho, \mu]$. A function $f \in \mathcal{A}$ is in the class $\mathcal{R}[\rho, \mu]$ if $f * \mathcal{S}_{\rho} \in \mathcal{S}^{*}(\mu)$, where $\mathcal{S}_{\rho}=\frac{z}{(1-z)^{2-2 \rho}}, 0 \leq \rho<1$. It is easy to see that $\mathcal{R}[\mu, \mu]=\mathcal{R}_{\mu}$. For more details, see $[8$, 29].

Observe that Mittag-Leffler function $\mathbb{E}_{\alpha, \beta}$ does not belong to the family $\mathcal{A}$. Thus, it is natural to consider the following normalization of Mittag-Leffler functions:

$$
\begin{gathered}
E_{\alpha, \beta}(z)=z \Gamma(\beta) \mathbb{E}_{\alpha, \beta}(z)=z+\sum_{n=2}^{\infty} \frac{\Gamma(\beta)}{\Gamma(\beta+\alpha(n-1))} z^{n}, \\
z, \alpha, \beta \in \mathbb{C}, \operatorname{Re}(\alpha)>0, \operatorname{Re}(\beta)>0 .
\end{gathered}
$$

Formula (1.4) holds for complex parameters $\alpha, \beta$, and $z \in \mathbb{C}$. In this paper, we shall restrict our attention to the case of real-valued $\alpha, \beta$, and $z \in \mathcal{U}$. For particular values of $\alpha$ and $\beta$, we obtain several functions, for example

$$
\begin{array}{ll}
E_{1,3}(z)=\frac{2\left(e^{z}-z-1\right)}{z}, & E_{1,4}(z)=\frac{6\left(e^{z}-z-1\right)-3 z^{2}}{z^{2}} \\
E_{2,2}(z)=\sqrt{z} \sinh \sqrt{z}, & E_{3,1}(z)=\frac{z}{2}\left[e^{z^{1 / 3}}+2 e^{-\frac{1}{2} z^{1 / 3}} \cos \left(\frac{\sqrt{3}}{2} z^{1 / 3}\right)\right] .
\end{array}
$$

In some recent years, several researchers studied geometric properties such as starlikeness, convexity, and close-to-convexity of certain special functions; for details, see [5-7, $19,21-25,36]$ and the references therein. Also see $[2,13,16,30,33]$ for some properties of special functions and mathematical inequalities. More recently, Sangal and Swaminathan [31] studied geometric properties of hypergeometric functions by using the positivity technique. 
In this paper, we study pre-starlikeness and deduce the convexity and starlikeness of order $1 / 2$. We also investigate the starlikeness of order $2 \mu+\eta-1$. Furthermore, we find the convexity of Mittag-Leffler functions in the direction of imaginary axis. The main tool in this investigation is the positivity technique.

\section{Preliminaries}

To obtain our main results, we need the following lemmas.

Lemma 2.1 ([18]) Let $\left\{a_{k}\right\}_{k=1}^{\infty}$ be a sequence of positive numbers such that $a_{1}=1$. If, for $0 \leq \mu<1$,

(1) $(1-\mu) a_{1} \geq(2-\mu) a_{2} \geq 2^{(\mu+1)}(3-\mu) a_{3}$,

(2) $(k-1-\mu)(k-\mu) a_{k} \geq k(k+1-\mu) a_{k+1}, \forall k \geq 3$.

Then $f(z)=z+\sum_{k=2}^{\infty} a_{k} z^{k} \in \mathcal{S}^{*}(\mu)$.

Lemma 2.2 ([20]) Let $\eta \geq 0, \mu \in \mathbb{R}$ such that $0<\mu+\eta<1$ and $n \in \mathbb{N}$. If $d_{0}=d_{1}=1$ and $d_{2 k}=d_{2 k+1}=\frac{(1+\eta)_{n-k} n !}{(n-k) !(1+\eta)_{n}} \cdot \frac{(\mu+\eta)_{k}}{k !}$ for $1 \leq k \leq n$, then

(i) $\sum_{k=0}^{n} d_{k} \cos (k \theta)>0 \Leftrightarrow \mu+\eta \leq \mu^{*}\left(\frac{1}{2}\right)=0.691556 \ldots$,

(ii) $\sum_{k=1}^{2 n+1} \sin (k \theta)>0 \Leftrightarrow \mu+\eta \leq \mu^{*}\left(\frac{1}{2}\right)$,

(iii) $\sum_{k=1}^{2 n} \sin (k \theta)>0$ for $\mu+\eta \leq \frac{1+\eta}{2}$,

where $\mu^{*}(\gamma), \gamma \in(0,1]$ is the unique solution in $] 0,1\left[\right.$ of $\int_{0}^{(\gamma+1) \pi} \frac{\sin (t-\gamma \pi)}{t^{1-\mu}} d t=0$.

Koumandos and Ruscheweyh [15] obtained the value of $\mu^{*}(\gamma)$. In this work, we use the particular value $\mu^{*}\left(\frac{1}{2}\right)=\mu_{0}^{*}$.

Lemma 2.3 ([31]) Let $0 \leq \eta \leq 2 \mu_{0}^{*}-1, \mu \in \mathbb{R}$ such that $0<\mu+\eta<1$ and $n \in \mathbb{N}$. If $\left\{a_{k}\right\}_{k=1}^{\infty}$ is a decreasing sequence of non-negative numbers satisfying $a_{0}>0$ and

$$
k(n-k+1+\eta) a_{2 k} \leq(n-k+1)(k+\mu+\eta-1) a_{2 k-1} \quad \text { for } 1 \leq k \leq n,
$$

then, for all $0<\theta<\pi$,

$$
\sum_{k=0}^{n} a_{k} \sin k \theta>0 \quad \Leftrightarrow \quad \mu+\eta \leq \frac{1+\eta}{2} .
$$

Lemma 2.4 ([31]) Let $0 \leq \eta \leq 2 \mu_{0}^{*}-1$ and $-\eta<\mu \leq \frac{1-\eta}{2}, a_{1}=1, a_{k} \geq 0$ satisfy

$$
\begin{aligned}
& {[k(1+\eta)(1-\mu-\eta)-1+2 \mu+\eta] a_{k}} \\
& \quad \geq[(k+1)(1+\eta)(1-\mu-\eta)-1+2 \mu+\eta] a_{k+1}, \\
& \quad(n-k+1)(k+\mu+\eta-1)[2 k(1+\eta)(1-\mu-\eta)-1+2 \mu+\eta] a_{2 k} \\
& \quad \geq k(n-k+1+\eta)[(2 k+1)(1+\eta)(1-\mu-\eta)-1+2 \mu+\eta] a_{2 k+1}
\end{aligned}
$$

for $1 \leq k \leq n$. Then $f_{n}(z)=\sum_{k=1}^{n} a_{k} z^{k}$ is starlike of order $\frac{1-2 \mu-\eta}{(1+\eta)(1-\mu-\eta)}$. Moreover, in the limiting case, $f(z)=\lim _{n \rightarrow \infty} f_{n}(z)=\sum_{k=1}^{\infty} a_{k} z^{k}$ is starlike of the same order if $\left\{a_{k}\right\}$ satisfy (2.1) and in addition

$$
\begin{aligned}
& (k+\mu+\eta-1)[2 k(1+\eta)(1-\mu-\eta)-1+2 \mu+\eta] a_{2 k} \\
& \quad \geq k[(2 k+1)(1+\eta)(1-\mu-\eta)-1+2 \mu+\eta] a_{2 k+1} \quad \text { for } k \geq 1 .
\end{aligned}
$$


Lemma 2.5 ([31]) Let $\mu \in \mathbb{R}$ and $\eta \geq 0$ such that $0<\mu+\eta<1$, and let $a_{1}=1$ and $a_{k} \geq 0$ satisfy

$$
\begin{aligned}
0 & \leq n a_{n} \leq \cdots \leq(k+1) a_{k+1} \leq k a_{k} \leq \cdots \leq 3 a_{3} \\
& \leq 2 a_{2} \leq \frac{\mu+\eta}{\mu_{0}^{*}}, \quad \mu+\eta \in\left(0, \mu_{0}^{*}\right],
\end{aligned}
$$

and

$$
2(n-k+1)(k+\mu+\eta-1) a_{2 k} \geq(2 k+1)(n-k+1+\eta) a_{2 k+1}, \quad 1 \leq k \leq\left[\frac{n}{2}\right] .
$$

Then $f_{n}(z)=z+\sum_{k=2}^{n} a_{k} z^{k}$ satisfies $\operatorname{Re}\left(f_{n}^{\prime}(z)\right)>1-\frac{\mu+\eta}{\mu_{0}^{*}}$.

\section{Main results}

Theorem 3.1 Let $\alpha \geq 1, \beta \geq 1$. Then $E_{\alpha, \beta}(z) \in \mathcal{R}[\rho, \mu]$ for $0 \leq \mu<1$ and

$$
\frac{\Gamma(\alpha+\beta)}{\Gamma(\beta)} \geq\left\{\begin{array}{l}
T_{1}(\rho, \mu), \quad 0 \leq \rho \leq \rho_{1}(\mu), \\
\max \left\{T_{1}(\rho, \mu), T_{2}(\rho, \mu) \frac{2 \Gamma^{2}(\alpha+\beta)}{\Gamma(\beta) \Gamma(2 \alpha+\beta)}, T_{3}(\rho, \mu) \frac{\Gamma(\alpha(k-1)+\beta)}{\Gamma(\alpha k+\beta)} \frac{\Gamma(\alpha+\beta)}{\Gamma(\beta)}\right\} \\
\quad \rho_{1}(\mu) \leq \rho<1
\end{array}\right.
$$

where

$$
\begin{array}{lc}
T_{1}(\rho, \mu)=\frac{2(2-\mu)(1-\rho)}{(1-\mu)}, & T_{2}(\rho, \mu)=\frac{2^{\mu-1}(3-\mu)(3-2 \rho)}{(2-\mu)}, \\
T_{3}(\rho, \mu)=\frac{2(4-\mu)(2-\rho)}{(2-\mu)(3-\mu)}, & \rho_{1}(\mu)=1-\frac{2^{\mu}(3-\mu)(1-\mu)}{4(2-\mu)^{2}-2.2^{\mu}(3-\mu)(1-\mu)} .
\end{array}
$$

Proof Consider the function $g(z)=z+\sum_{k=2}^{\infty} b_{k} z^{k}$, where $b_{k}$ is given by

$$
b_{1}=1, \quad b_{k+1}=\frac{\Gamma(\alpha(k-1)+\beta)}{\Gamma(\alpha k+\beta)} \frac{(k+1-2 \rho)}{k} b_{k}, \quad \forall k \geq 1 .
$$

Now

$$
\begin{aligned}
(1-\mu) b_{1}-(2-\mu) b_{2} \\
\quad=(1-\mu)-2(2-\mu) \frac{\Gamma(\beta)}{\Gamma(\alpha+\beta)}(1-\rho) \\
\quad=\frac{1}{\Gamma(\alpha+\beta)}[(1-\mu) \Gamma(\alpha+\beta)-2(2-\mu)(1-\rho) \Gamma(\beta)] \geq 0
\end{aligned}
$$

implies $\Gamma(\alpha+\beta) \geq T_{1}(\rho, \mu) \Gamma(\beta)$, where $T_{1}(\rho, \mu)=\frac{2(2-\mu)(1-\rho)}{(1-\mu)}$. Again

$$
\begin{aligned}
(2 & -\mu) b_{2}-2^{\mu+1}(3-\mu) b_{3} \\
& =(2-\mu) b_{2}-2^{\mu+1}(3-\mu) \frac{\Gamma(\alpha+\beta)}{\Gamma(2 \alpha+\beta)} \frac{(3-2 \rho)}{2} b_{2} \\
& =\frac{2(2-\mu) b_{2}}{\Gamma(2 \alpha+\beta)}\left[\frac{\Gamma(2 \alpha+\beta)}{2}-T_{2}(\rho, \mu) \Gamma(\alpha+\beta)\right] \geq 0
\end{aligned}
$$


with $\frac{\Gamma(\alpha+\beta)}{\Gamma(\beta)} \geq 2 T_{2}(\rho, \mu) \frac{\Gamma^{2}(\alpha+\beta)}{\Gamma(\beta) \Gamma(2 \alpha+\beta)}$, where $T_{2}(\rho, \mu)=\frac{2^{\mu-1}(3-\mu)(3-2 \alpha)}{(2-\mu)}$. Also consider

$$
\begin{aligned}
(k- & 1-\mu)(k-\mu) b_{k}-k(k+1-\mu) b_{k+1} \\
= & (k-1-\mu)(k-\mu) b_{k}-k(k+1-\mu) \frac{(k+1-2 \rho)}{k} \frac{\Gamma(\alpha(k-1)+\beta)}{\Gamma(\alpha k+\beta)} b_{k} \\
= & \frac{b_{k}}{\Gamma(\alpha k+\beta)} \\
& \quad \times[(k-1-\mu)(k-\mu) \Gamma(\alpha k+\beta)-(k+1-\mu)(k+1-2 \rho) \Gamma(\alpha(k-1)+\beta)] \\
= & A(k) M(k),
\end{aligned}
$$

where $A(k)=\frac{b_{k}}{\Gamma(\alpha k+\beta)}$ and

$$
\begin{aligned}
M(k) & =(k-1-\mu)(k-\mu) \Gamma(\alpha k+\beta)-(k+1-\mu)(k+1-2 \rho) \Gamma(\alpha(k-1)+\beta) \\
& =A(\rho, \mu)(k-3)^{2}+B(\rho, \mu)(k-3)+D(\rho, \mu) .
\end{aligned}
$$

Here

$$
\begin{aligned}
& A(\rho, \mu)=\Gamma(\alpha k+\beta)-\Gamma(\alpha(k-1)+\beta) \geq 0, \\
& B(\rho, \mu)=(5-2 \mu) \Gamma(\alpha k+\beta)-(8-2 \rho-\mu) \Gamma(\alpha(k-1)+\beta) \geq 0, \\
& D(\rho, \mu)=(2-\mu)(3-\mu) \Gamma(\alpha k+\beta)-2(4-\mu)(2-\rho) \Gamma(\alpha(k-1)+\beta) \geq 0 .
\end{aligned}
$$

This implies that

$$
\frac{\Gamma(\alpha+\beta)}{\Gamma(\beta)} \geq T_{3}(\rho, \mu) \frac{\Gamma(\alpha(k-1)+\beta)}{\Gamma(\alpha k+\beta)} \frac{\Gamma(\alpha+\beta)}{\Gamma(\beta)},
$$

where $T_{3}(\rho, \mu)=\frac{2(4-\mu)(2-\alpha)}{(2-\mu)(3-\mu)}$. It is clear that $A(\rho, \mu), B(\rho, \mu), D(\rho, \mu)$ are non-negative. Since each coefficient of $(k-3)$ and the constant term in $M(k)$ are non-negative, therefore $M(k)$ is an increasing function for $k \geq 3$. Also, for $M(3)>0$, we have $(k-1-\mu)(k-\mu) b_{k} \geq$ $k(k+1-\mu) b_{k+1}$. Thus $b_{k}$ satisfies the conditions of Lemma 2.1 and hence $g \in \mathcal{S}^{*}(\mu)$. After simple computations, we observe that $g(z)=E_{\alpha, \beta}(z) * \frac{z}{(1-z)^{2-2 \rho}}$. Therefore, by the definition of $\mathcal{R}[\rho, \mu]$, we have $E_{\alpha, \beta}(z) \in \mathcal{R}[\rho, \mu]$. Now consider

$$
\begin{aligned}
T_{3}(\rho, \mu)-T_{1}(\rho, \mu) & =\frac{2(4-\mu)(2-\rho)}{(2-\mu)(3-\mu)}-\frac{2(2-\mu)(1-\rho)}{(1-\mu)} \\
& =\frac{2(1-\mu)(4-\mu)(2-\rho)-2(2-\mu)^{2}(3-\mu)(1-\rho)}{(1-\mu)(2-\mu)(3-\mu)} .
\end{aligned}
$$

The numerator is negative for all $\mu$ and hence $T_{3}(\rho, \mu) \leq T_{1}(\rho, \mu)$ for $0 \leq \rho \leq \rho_{0}(\mu)$. Similarly, if $0 \leq \rho \leq \rho_{1}(\mu), T_{1}(\rho, \mu) \geq T_{2}(\rho, \mu)$ for all $\mu$. Here,

$$
\begin{aligned}
& \rho_{0}(\mu)=1-\frac{(4-\mu)(1-\mu)}{(2-\mu)^{2}(3-\mu)-(4-\mu)(1-\mu)}, \\
& \rho_{1}(\mu)=1-\frac{2^{\mu}(3-\mu)(1-\mu)}{4(2-\mu)^{2}-2.2^{\mu}(3-\mu)(1-\mu)} .
\end{aligned}
$$


Clearly, we can investigate that, for $0 \leq \rho \leq \min \left\{\rho_{0}(\mu), \rho_{1}(\mu)\right\}$,

$$
\max _{i=1,2,3}\left\{T_{i}(\rho, \mu)\right\}=T_{1}(\rho, \mu)
$$

Now, we only need to check the $\min \left\{\rho_{0}(\mu), \rho_{1}(\mu)\right\}$. Consider

$$
\begin{aligned}
\rho_{1}-\rho_{0} & =-\frac{2^{\mu}(3-\mu)(1-\mu)}{4(2-\mu)^{2}-2.2^{\mu}(3-\mu)(1-\mu)}+\frac{(4-\mu)(1-\mu)}{(2-\mu)^{2}(3-\mu)-(4-\mu)(1-\mu)} \\
& =\frac{N(\mu)}{\left(4(2-\mu)^{2}-2.2^{\mu}(3-\mu)(1-\mu)\right)\left((2-\mu)^{2}(3-\mu)-(4-\mu)(1-\mu)\right)},
\end{aligned}
$$

where

$$
\begin{aligned}
N(\mu)= & 2^{\mu}(3-\mu)(1-\mu)\left\{(2-\mu)^{2}(3-\mu)-(4-\mu)(1-\mu)\right\} \\
& +(4-\mu)(1-\mu)\left\{4(2-\mu)^{2}-2.2^{\mu}(3-\mu)(1-\mu)\right\} \\
< & 0 .
\end{aligned}
$$

This implies $\rho_{0}(\mu)=\min \left\{\rho_{0}(\mu), \rho_{1}(\mu)\right\}$, and the proof is complete.

Theorem 3.2 Let $0 \leq \mu<1, \alpha \geq 1, \beta \geq 1$. If $\frac{\Gamma(\alpha+\beta)}{\Gamma(\beta)} \geq 2(2-\mu)$, then $E_{\alpha, \beta}(z)$ is pre-starlike of order $\mu$ in $\mathcal{U}$.

Proof Consider $T_{i}(\rho, \mu), i=1,2,3$, as in Theorem 3.1. Replacing $\rho$ by $\mu$, we get

$$
\begin{aligned}
& T_{1}(\mu)=2(2-\mu), \\
& T_{2}(\mu)=\frac{2^{\mu-1}(3-\mu)(3-2 \mu)}{(2-\mu)}, \\
& T_{3}(\mu)=\frac{2(4-\mu)}{(3-\mu)} .
\end{aligned}
$$

It is noticed that, for $0 \leq \mu<1$,

$$
\begin{aligned}
T_{1}(\mu)-T_{2}(\mu) & =2(2-\mu)-\frac{2^{\mu-1}(3-\mu)(3-2 \mu)}{(2-\mu)} \\
& =\frac{2(2-\mu)^{2}-2^{\mu-1}(3-\mu)(3-2 \mu)}{(2-\mu)}>0 .
\end{aligned}
$$

Similarly,

$$
\begin{aligned}
T_{1}(\mu)-T_{3}(\mu) & =2(2-\mu)-\frac{2(4-\mu)}{(3-\mu)} \\
& =\frac{2(2-\mu)(3-\mu)-2(4-\mu)}{(3-\mu)}>0 .
\end{aligned}
$$

Therefore, $T_{1}(\mu)$ is maximum. Hence

$$
\frac{\Gamma(\alpha+\beta)}{\Gamma(\beta)} \geq \max \left\{\begin{array}{c}
T_{1}(\mu), T_{2}(\mu) \frac{2 \Gamma^{2}(\alpha+\beta)}{\Gamma(\beta) \Gamma(2 \alpha+\beta)}, \\
T_{3}(\mu) \frac{\Gamma(\alpha(k-1)+\beta)}{\Gamma(\alpha k+\beta)} \frac{\Gamma(\alpha+\beta)}{\Gamma(\beta)}
\end{array}\right\}=T_{1}(\mu)
$$


This is equivalent to

$$
\frac{\Gamma(\alpha+\beta)}{\Gamma(\beta)} \geq 2(2-\mu) .
$$

Corollary 3.3 Let $\alpha \geq 1, \beta \geq 1$. Then $E_{\alpha, \beta}(z) \in \mathcal{C}$ if $\frac{\Gamma(\alpha+\beta)}{\Gamma(\beta)} \geq 4$.

Proof It is noticed that, for $\mu=0$, we have $\frac{z}{(1-z)^{2}} * f(z) \in \mathcal{S}^{*}$. By using the definition of convolution, it is easy to see that $z f^{\prime}(z) \in \mathcal{S}^{*}$. Therefore, by Alexander relation it follows that $f \in \mathcal{C}$. We also see from Theorem 3.2 that

$$
T_{1}(0)=4, \quad T_{2}(0)=\frac{9}{4}, \quad T_{3}(0)=\frac{8}{3} .
$$

Now

$$
T_{1}(0)-T_{2}(0)=4-\frac{9}{4}=\frac{7}{4}>0,
$$

similarly,

$$
T_{1}(0)-T_{3}(0)=4-\frac{8}{3}=\frac{4}{3}>0 .
$$

Therefore, $T_{1}(0)$ is maximum. Hence

$$
\frac{\Gamma(\alpha+\beta)}{\Gamma(\beta)} \geq 4
$$

This shows that $E_{\alpha, \beta}(z) \in \mathcal{C}$.

Example 3.4 For $\alpha=3, \beta=1$, we have $\frac{\Gamma(\alpha+\beta)}{\Gamma(\beta)} \geq 4$, therefore the function

$$
E_{3,1}(z)=\frac{z}{2}\left[e^{z^{1 / 3}}+2 e^{-\frac{1}{2} z^{1 / 3}} \cos \left(\frac{\sqrt{3}}{2} z^{1 / 3}\right)\right]
$$

is in $\mathcal{C}$.

Example 3.5 For $\alpha=1, \beta=4$, we have $\frac{\Gamma(\alpha+\beta)}{\Gamma(\beta)}=4$, therefore the function

$$
E_{1,4}(z)=\frac{6\left(e^{z}-z-1\right)-3 z^{2}}{z^{2}}
$$

is in $\mathcal{C}$.

The mappings of these functions are given in Fig. 1. 


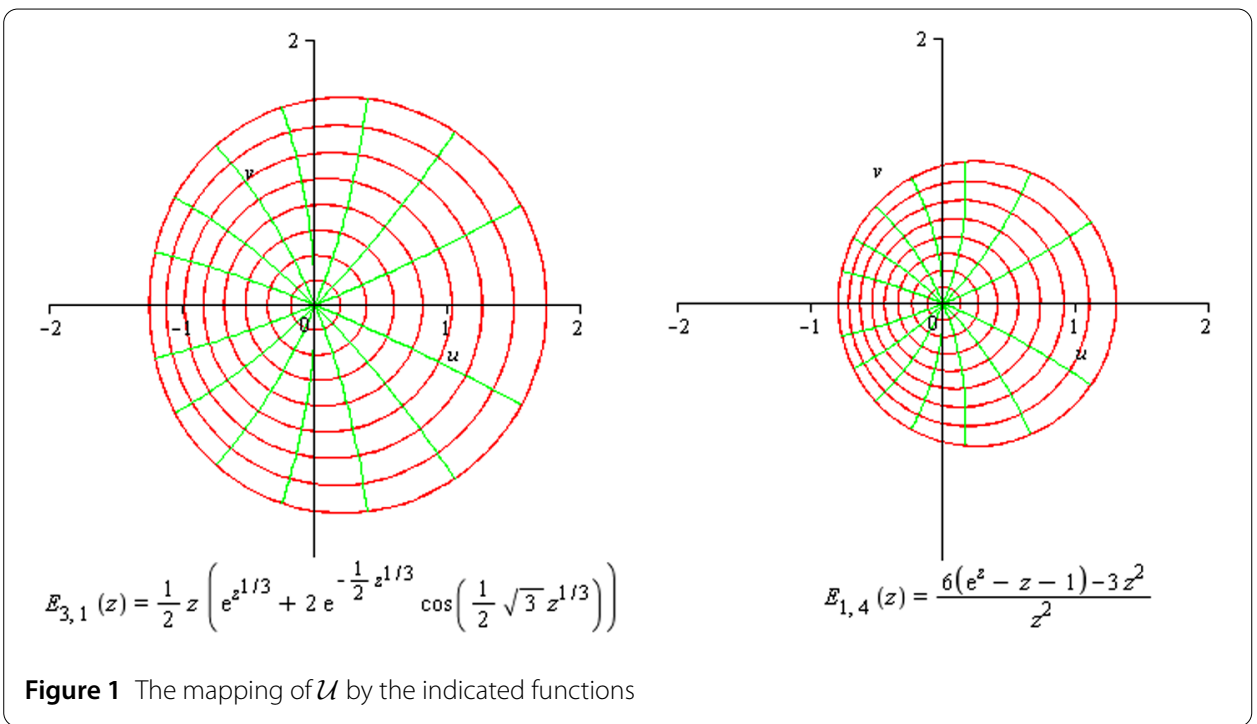

Corollary 3.6 Let $\alpha \geq 1, \beta \geq 1$. Then $E_{\alpha, \beta}(z) \in \mathcal{S}^{*}\left(\frac{1}{2}\right)$ if $\frac{\Gamma(\alpha+\beta)}{\Gamma(\beta)} \geq 3$.

Proof Consider $T_{i}(\rho, \mu), i=1,2,3$, as in Theorem 3.1. Replacing $\rho$ by $\mu$, we get

$$
\begin{aligned}
& T_{1}(\mu)=2(2-\mu), \\
& T_{2}(\mu)=\frac{2^{\mu-1}(3-\mu)(3-2 \mu)}{(2-\mu)}, \\
& T_{3}(\mu)=\frac{2(4-\mu)}{(3-\mu)} .
\end{aligned}
$$

For $\mu=\frac{1}{2}$,

$$
T_{1}\left(\frac{1}{2}\right)=3, \quad T_{2}\left(\frac{1}{2}\right)=\frac{5 \sqrt{2}}{3}, \quad T_{3}\left(\frac{1}{2}\right)=\frac{14}{5} .
$$

Now

$$
T_{1}\left(\frac{1}{2}\right)-T_{2}\left(\frac{1}{2}\right)=3-\frac{5 \sqrt{2}}{3}=\frac{9-5 \sqrt{2}}{3}>0 .
$$

Similarly,

$$
T_{1}\left(\frac{1}{2}\right)-T_{3}\left(\frac{1}{2}\right)=3-\frac{14}{5}=\frac{1}{5}>0 .
$$

Therefore, $T_{1}\left(\frac{1}{2}\right)$ is maximum. Hence

$$
\frac{\Gamma(\alpha+\beta)}{\Gamma(\beta)} \geq 3 .
$$

Therefore, for $\alpha \geq 1, \beta \geq 1$, and $\frac{\Gamma(\alpha+\beta)}{\Gamma(\beta)} \geq 3, E_{\alpha, \beta}(z) \in \mathcal{S}^{*}\left(\frac{1}{2}\right)$. 




Figure 2 The mapping of $\mathcal{U}$ by the indicated functions

Example 3.7 For $\alpha=1, \beta=3$, we have $\frac{\Gamma(\alpha+\beta)}{\Gamma(\beta)}=3$, therefore the function

$$
E_{1,3}(z)=\frac{2\left(e^{z}-z-1\right)}{z}
$$

is in $\mathcal{S}^{*}\left(\frac{1}{2}\right)$.

Example 3.8 For $\alpha=2, \beta=2$, we have $\frac{\Gamma(\alpha+\beta)}{\Gamma(\beta)}=6$, therefore the function

$$
E_{2,2}(z)=\sqrt{z} \sinh \sqrt{z}
$$

is in $\mathcal{S}^{*}\left(\frac{1}{2}\right)$.

The mappings of these functions are given in Fig. 2.

Theorem 3.9 Let $\mu \geq 1, \eta \geq \frac{-\mu+\sqrt{\mu^{2}+4 \mu-4}}{2}$ with $\alpha \geq 1, \beta \geq 2$ If $M_{1}=(1+\eta)(1-\mu-\eta)>0$ and $M_{2}=+2 \mu+\eta-1>0$, then $E_{\alpha, \beta}(z)$ is starlike of order $2 \mu+\eta-1$.

Proof It is observed that $\left(E_{\alpha, \beta}\right)_{n}(z)=\sum_{k=1}^{\infty} a_{k} z^{k}$ gives $a_{1}=1$ and $a_{k}=\frac{\Gamma(\beta)}{\Gamma(\alpha(k-1)+\beta)}$ for $k \geq 2$. The relation between $a_{k}$ and $a_{k+1}$ is

$$
a_{k+1}=\frac{\Gamma(\alpha(k-1)+\beta)}{\Gamma(\alpha k+\beta)} a_{k} \quad \text { for } k \geq 1
$$

To prove this theorem, it is enough to show that $\left\{a_{k}\right\}$ satisfies conditions (2.1) and (2.3) of Lemma 2.4. Using the above relation and simple computations yields

$$
\begin{aligned}
& {[k(1+\eta)(1-\mu-\eta)-1+2 \mu+\eta] a_{k}-[(k+1)(1+\eta)(1-\mu-\eta)-1+2 \mu+\eta] a_{k+1}} \\
& \quad=\frac{\Gamma(\alpha(k-1)+\beta) a_{k}}{\Gamma(\alpha k+\beta)} h(k),
\end{aligned}
$$


where $h(k)$ is defined as follows:

$$
\begin{aligned}
h(k)= & \frac{\Gamma(\alpha k+\beta)}{\Gamma(\alpha(k-1)+\beta)}[k(1+\eta)(1-\mu-\eta)-1+2 \mu+\eta] \\
& -[(k+1)(1+\eta)(1-\mu-\eta)-1+2 \mu+\alpha] \\
= & \frac{\Gamma(\alpha k+\beta)}{\Gamma(\alpha(k-1)+\beta)}\left(k M_{1}+M_{2}\right)-\left[(k+1) M_{1}+M_{2}\right] \\
= & {\left[\frac{k \Gamma(\alpha k+\beta)}{\Gamma(\alpha(k-1)+\beta)}-(k+1)\right] M_{1}+\left[\frac{\Gamma(\alpha k+\beta)}{\Gamma(\alpha(k-1)+\beta)}-1\right] M_{2}>0 . }
\end{aligned}
$$

It is observed that under the conditions $\mu \geq 1, \eta \geq \frac{-\mu+\sqrt{\mu^{2}+4 \mu-4}}{2}, \alpha \geq 1$, and $\beta \geq 3$, expression (3.2) is positive for $k \geq 1$. It remains to verify (2.3). That is,

$$
\begin{array}{r}
(k+\mu+\eta-1)[2 k(1+\eta)(1-\mu-\eta)-1+2 \mu+\eta] a_{2 k} \\
\quad \geq k[(2 k+1)(1+\eta)(1-\mu-\eta)-1+2 \mu+\eta] a_{2 k+1} .
\end{array}
$$

Clearly,

$$
\begin{aligned}
& (k+\mu+\eta-1)[2 k(1+\eta)(1-\mu-\eta)-1+2 \mu+\eta] a_{2 k} \\
& \quad-k[(2 k+1)(1+\eta)(1-\mu-\eta)-1+2 \mu+\eta] a_{2 k+1}=\frac{\Gamma(\alpha(2 k-1)+\beta) a_{2 k}}{2 \Gamma(\alpha(2 k)+\beta)} g(k),
\end{aligned}
$$

where $g(k)$ is defined as follows:

$$
\begin{aligned}
g(k)= & \left.\frac{\Gamma(\alpha(2 k)+\beta)}{\Gamma(\alpha(2 k-1)+\beta)}\left(k+\mu+\eta^{\prime}-1\right)[2 k(1+\eta)(1-\mu-\eta)-1+2 \mu+\eta)\right] \\
& -[(2 k+1)(1+\eta)(1-\mu-\eta)-1+2 \mu+\eta] \\
= & \frac{\Gamma(\alpha(2 k)+\beta)}{\Gamma(\alpha(2 k-1)+\beta)}(k+\mu+\eta-1)\left[2 k M_{1}+M_{2}\right]-k\left[(2 k+1) M_{1}+M_{2}\right] \\
= & {\left[\frac{\Gamma(\alpha(2 k)+\beta)}{\Gamma(\alpha(2 k-1)+\beta)} 2 k(k+\mu+\eta-1)-k(2 k+1)\right] M_{1} } \\
& +\left[\frac{\Gamma(\alpha(2 k)+\beta)}{\Gamma(\alpha(2 k-1)+\beta)}(k+\mu+\eta-1)-k\right] M_{2}>0 .
\end{aligned}
$$

It is observed that under the conditions $\mu \geq 1, \eta \geq \frac{-\mu+\sqrt{\mu^{2}+4 \mu-4}}{2}, 2 \mu+\eta>1, \alpha \geq 1$, and $\beta \geq 2$, expression (3.3) is positive for $k \geq 1$, which completes the proof.

Theorem 3.10 Let $\mu \geq 1, \eta \geq \frac{-\mu+\sqrt{\mu^{2}+4 \mu-4}}{2}, 2 \mu+\eta>1, \alpha \geq 1, \beta \geq 2, a_{1}=1, a_{k} \geq 0$ satisfy

$$
\begin{aligned}
& k a_{k}-(k+1) a_{k+1} \geq 0, \quad k=1,2,3, \ldots, n-1, \\
& (n-k+1)(k+\mu+\eta-1)(2 k-1) a_{2 k-1} \geq 2 k^{2}(n-k+1+\eta) a_{2 k}, \\
& \quad k=4,5, \ldots,\left[\frac{n+3}{2}\right],
\end{aligned}
$$

for $k \geq 4$. Then $\left(E_{\alpha, \beta}\right)_{n}(z)=\sum_{k=4}^{n} a_{k} z^{k}$ is convex in the direction of imaginary axis. 
Proof To show that Mittag-Leffler function is convex in the direction of imaginary axis, we will prove that $z\left(E_{\alpha, \beta}\right)_{n}^{\prime}(z)$ is a typically real function. Also $\left(E_{\alpha, \beta}\right)_{n}(z)$ has real coefficients. Set

$$
\begin{aligned}
z\left(E_{\alpha, \beta}\right)_{n}^{\prime}(z) & =z+\sum_{k=2}^{n} \frac{\Gamma(\beta)}{\Gamma(\alpha(k-1)+\beta)} z^{k} \\
& =z+\sum_{k=2}^{n} b_{k} z^{k},
\end{aligned}
$$

where $b_{k}=\frac{\Gamma(\beta)}{\Gamma(\alpha(k-1)+\beta)}$. To get the result, it is required that $\left\{b_{k}\right\}$ must satisfy the conditions mentioned in Lemma 2.3. Consider

$$
\begin{aligned}
k b_{k}-(k+1) b_{k+1} & =\Gamma(\beta)\left[\frac{k}{\Gamma(\alpha(k-1)+\beta)}-\frac{k+1}{\Gamma(\alpha k+\beta)}\right] \\
& =\Gamma(\beta)\left[\frac{k \Gamma(\alpha k+\beta)-(k+1) \Gamma(\alpha(k-1)+\beta)}{\Gamma(\alpha k+\beta) \Gamma(\alpha(k-1)+\beta)}\right]>0
\end{aligned}
$$

for $k=1,2,3, \ldots, n-1$. Take

$$
(n-k+1)(k+\mu+\eta-1) b_{2 k-1}-k(n-k+1+\eta) b_{2 k}=\frac{b_{2 k} \Gamma(\alpha(2 k-1)+\beta)}{\Gamma(\alpha(2 k-2)+\beta)} q(k),
$$

here $q(k)$ is defined as

$$
q(k)=(k+\mu+\eta-1)(n-k+1)-k(n-k+1+\eta) \frac{\Gamma(\alpha(2 k-2)+\beta)}{\Gamma(\alpha(2 k-1)+\beta)} .
$$

Since $\Gamma$ is an increasing function in $\left[\frac{3}{2}, \infty\right)$, therefore (3.5) becomes positive when $\mu \geq$ $1, \eta \geq \frac{-\mu+\sqrt{\mu^{2}+4 \mu-4}}{2}, 2 \mu+\eta>1, \alpha \geq 1$, and $\beta \geq 2$. Thus $\left\{b_{k}\right\}$ satisfies the conditions of Lemma 2.3. Therefore, by using the minimum principle for harmonic functions under the conditions $\mu+\eta \in\left(0, \frac{1+\eta}{2}\right]$,

$$
\left.\operatorname{Im}\left(z f_{n}^{\prime}(z)\right)=\sum_{k=1}^{n} b_{k} r^{k} \sin k \theta>0, \quad \text { where } \theta \in\right] 0, \pi[\text { and } r \in] 0,1[
$$

and

$$
\operatorname{Im}\left(z f_{n}^{\prime}(z)\right)=0 \quad \text { for } z \in(0,1)
$$

The Schwarz reflection principle yields that $\operatorname{Im}\left(z f_{n}^{\prime}(z)\right)<0$ for $\theta \in(\pi, 2 \pi)$. So $z f_{n}^{\prime}(z)$ is a typically real function, which is equivalent to saying that $f_{n}(z)$ is convex in the direction of imaginary axis.

Theorem 3.11 Let $\mu \in \mathbb{R}$ and $\eta \geq 0$ such that $2 \mu+\eta>1$, and let $a_{1}=1$ and $a_{k} \geq 0$ satisfy

$$
0 \leq n a_{n} \leq \cdots \leq(k+1) a_{k+1} \leq k a_{k} \leq \cdots \leq 3 a_{3} \leq 2 a_{2} \leq \frac{\mu+\eta}{\mu_{0}^{*}}, \quad \mu+\eta \in\left(0, \mu_{0}^{*}\right]
$$


and

$$
2(n-k+1)(k+\mu+\eta-1) a_{2 k} \geq(2 k+1)(n-k+1+\eta) a_{2 k+1}, \quad 1 \leq k \leq\left[\frac{n}{2}\right] .
$$

If $\alpha \geq 1$ and $\beta \geq 2$, then $\left(E_{\alpha, \beta}\right)_{n}(z)=z+\sum_{k=2}^{n} a_{k} z^{k}$ satisfies $\operatorname{Re}\left(f_{n}^{\prime}(z)\right)>1-\frac{\mu+\eta}{\mu_{0}^{*}}$.

Proof Let $\sigma-1=-\frac{\mu+\eta}{\mu_{0}^{*}}$ and $\left(E_{\alpha, \beta}\right)_{n}(z)=z+\sum_{k=2}^{n} \frac{\Gamma(\beta)}{\Gamma(\alpha(k-1)+\beta)} z^{k}$, where $a_{k}=\frac{\Gamma(\beta)}{\Gamma(\alpha(k-1)+\beta)}$. Then

$$
\frac{\left(E_{\alpha, \beta}\right)_{n}^{\prime}(z)-\sigma}{1-\sigma}=\sum_{k=0}^{n-1} c_{k} z^{k}
$$

where $c_{k}=\frac{(k+1) b_{k+1}}{1-\sigma}$ and $c_{0}=1$ for $1 \leq k \leq n-1$. It is observed that under the conditions $\alpha \geq 1$ and $\beta \geq 2$ the coefficients $a_{k}$ are positive. Therefore, $c_{k}>0$ for $k \geq 1$. To prove this theorem, we will show that the coefficients $\left\{c_{k}\right\}$ are decreasing and satisfy (2.5). Now, for this, consider

$$
\begin{aligned}
(k+1) a_{k+1}-(k+2) a_{k+2} & =\Gamma(\beta)\left[\frac{k+1}{\Gamma(\alpha k+\beta)}-\frac{k+2}{\Gamma(\alpha(k+1)+\beta)}\right] \\
& =\Gamma(\beta)\left[\frac{(k+1) \Gamma(\alpha(k+1)+\beta)-(k+2) \Gamma(\alpha k+\beta)}{\Gamma(\alpha k+\beta) \Gamma(\alpha(k+1)+\beta)}\right]>0
\end{aligned}
$$

for $k=1,2,3, \ldots, n-2$. This shows that the coefficients of Mittag-Leffler function are decreasing and $c_{1}<c_{0} \Rightarrow 2 b_{2}<1-\sigma$. Now to have (2.5), consider

$$
\begin{aligned}
(n- & k+1)(\mu+\eta+k-1) a_{2 k}-(n-k+1+\eta)(2 k+1) a_{2 k+1} \\
= & \frac{(n-k+1)(\mu+\eta+k-1) \Gamma(\beta)}{\Gamma(\alpha(2 k-1)+\beta)}-\frac{(n-k+1+\eta)(2 k+1) \Gamma(\beta)}{\Gamma(\alpha(2 k)+\beta)} \\
= & \Gamma(\beta) \\
& \times\left[\frac{(n-k+1)(\mu+\eta+k-1) \Gamma(\alpha(2 k)+\beta)-(n-k+1+\eta)(2 k+1) \Gamma(\alpha(2 k-1)+\beta)}{\Gamma(\alpha(2 k)+\beta) \Gamma(\alpha(2 k-1)+\beta)}\right] \\
>0 & \text { for } 1 \leq k \leq\left[\frac{n}{2}\right] .
\end{aligned}
$$

It is clear that the above relation is positive for $n \geq k, \alpha \geq 1$, and $\beta \geq 2$. Also, $\Gamma$ is an increasing function in $\left[\frac{3}{2}, \infty\right)$. This yields (3.7). Using similar arguments and the minimum principle for harmonic function gives the required result.

\section{Conclusion}

In this paper, we have studied the normalized Mittag-Leffler function of two parameters. We have investigated new properties including pre-starlikeness, convexity, and starlikeness of order $1 / 2$. Sufficient conditions for the normalized Mittag-Leffler function to be starlike of order $2 \mu+\eta-1$ have also been studied. Moreover, we have found the convexity of Mittag-Leffler functions in the direction of imaginary axis. 
Availability of data and materials

Not applicable.

\section{Competing interests}

The authors declare that they have no competing interests.

\section{Authors' contributions}

MR and SN came up with the main thoughts, SN proved the main theorems, SNM reviewed them. All authors read and approved the final manuscript.

\section{Author details}

'Department of Mathematics, Government College University Faisalabad, Faisalabad, Pakistan. ${ }^{2}$ Department of Mathematics, COMSATS University Islamabad, Wah Campus, Pakistan.

\section{Publisher's Note}

Springer Nature remains neutral with regard to jurisdictional claims in published maps and institutional affiliations.

Received: 3 December 2018 Accepted: 26 March 2019 Published online: 03 April 2019

\section{References}

1. Agarwal, P., Al-Mdallal, Q., Cho, Y.J., Jain, S.: Fractional differential equations for the generalized Mittag-Leffler function Adv. Differ. Equ. 2018, 58 (2018)

2. Agarwal, P., Dragomir, S.S., Jleli, M., Samet, B.: Advances in Mathematical Inequalities and Applications, 1 st edn. Birkhäuser, Basel (2018)

3. Agarwal, P., Nieto, J.J.: Some fractional integral formulas for the Mittag-Leffler type function with four parameters. Open Math. 13, 537-546 (2015)

4. Agarwal, R.P.: A propos d'une note de M. Pierre Humbert. C. R. Acad. Sci. Paris 236, 2031-2032 (1953)

5. Bansal, D., Prajapat, J.K.: Certain geometric properties of the Mittag-Leffler functions. Complex Var. Elliptic Equ. 61(3), $338-350$ (2016)

6. Baricz, Á.: Generalized Bessel Functions of the First Kind. Lecture Notes in Mathematics, vol. 1994. Springer, Berlin (2010)

7. Baricz, Á., Ponnusamy, S.: Starlikeness and convexity of generalized Bessel functions. Integral Transforms Spec. Funct. 21(9), 641-653 (2010)

8. Duren, P.L.: Univalent Functions. Springer, New York (1983)

9. Gorenflo, R., Kilbas, A.A., Mainardi, F., Rogosin, S.V.: Mittag-Leffler Functions, Related Topics and Applications. Springer Monogr. Math. Springer, Heidelberg (2014)

10. Humbert, P.: Quelques resultants retifs a la fonction de Mittag-Leffler. Comptes Rendus de L'Academie Des Sciences $236,1467-1468(1953)$

11. Humbert, P., Agarwal, R.P.: Sur la fonction de Mittag-Leffler et quelques unes de ses generalizations. Bull. Sci. Math Ser. II 77, 180-185 (1953)

12. Jain, S., Agarwal, P., Onur Kiymaz, I., Cetinkaya, A.: Some composition formulae for the MSM fractional integral operator with the multi-index Mittag-Leffler functions. AIP Conf. Proc. 1926, 020020 (2018). https://doi.org/10.1063/1.5020469

13. Jain, S., Mehrez, K., Baleanu, D., Agarwal, P.: Certain Hermite-Hadamard inequalities for logarithmically convex functions with applications. Mathematics 7, 163 (2019)

14. Kilbas, A.A., Srivastava, H.M., Trujillo, J.J.: Theory and Applications of Fractional Differential Equations. North-Holland Mathematics Studies, vol. 204. Elsevier, Amsterdam (2006)

15. Koumandos, S., Ruscheweyh, S.: On a conjecture for trigonometric sums and starlike functions. J. Approx. Theory 149(1), 42-58 (2007)

16. Mehrez, K., Agarwal, P.: New Hermite-Hadamard type integral inequalities for convex functions and their applications. J. Comput. Appl. Math. 350, 274-285 (2019)

17. Mittag-Leffler, G.M.: Sur la nouvelle fonction $E_{\alpha}$ X. C. R. Acad. Sci. Paris 137, 554-558 (1903)

18. Mondal, S.R., Swaminathan, A.: On the positivity of certain trigonometric sums and their applications. Comput. Math. Appl. 62(10), 3871-3883 (2011)

19. Mondal, S.R., Swaminathan, A.: Geometric properties of generalized Bessel functions. Bull. Malays. Math. Sci. Soc 35(1), 179-194 (2012)

20. Mondal, S.R., Swaminathan, A.: Stable functions and extension of Vietoris' theorem. Results Math. 62(1-2), 33-51 (2012)

21. Orhan, H., Yağmur, N.: Geometric properties of generalized Struve functions. An. Ştiinţ. Univ. 'Al.I. Cuza' laşi, Mat. https://doi.org/10.2478/aicu-2014-0007

22. Prajapat, J.K.: Certain geometric properties of the Wright function. Integral Transforms Spec. Funct. 26(3), 203-212 (2015)

23. Răducanu, D.: On partial sums of normalized Mittag-Leffler functions. An. Ştiinţ. Univ. 'Ovidius' Constanţa, Ser. Mat. 25(2), 123-133 (2017)

24. Răducanu, D.: Third-order differential subordinations for analytic functions associated with generalized Mittag-Leffler functions. Mediterr. J. Math. 14(4), 167 (2017)

25. Raza, M., Din, M.U., Malik, S.N.: Certain geometric properties of normalized Wright functions. J. Funct. Spaces 2016 Article ID 1896154 (2016)

26. Robertson, M.S.: On the theory of univalent functions. Ann. Math. 37, 374-408 (1936)

27. Ruscheweyh, S.: Coefficient conditions for starlike functions. Glasg. Math. J. 29, 141-142 (1987)

28. Ruscheweyh, St.: Convolutions in Geometric Function Theory. Les Presses De L'Universite De Montreal, Montreal (1982) 
29. Ruscheweyh, St., Sheil-Small, T.: Hadamard products of Schlicht functions and the Pólya-Schoenberg conjecture. Comment. Math. Helv. 48(1), 119-135 (1973)

30. Ruzhansky, M.V., Je Cho, Y., Agarwal, P., Area, I.: Advances in Real and Complex Analysis with Applications. Springer, Singapore (2017)

31. Sangal, P., Swaminathan, A.: Starlikeness of Gaussian hypergeometric functions using positivity techniques. Bull. Malays. Math. Sci. Soc. (2016). https://doi.org/10.1007/s40840-016-0420-5

32. Sheil-Small, T., Silverman, H., Silvia, E.: Convolution multipliers and starlike functions. J. Anal. Math. 41, 181-192 (1982)

33. Sitho, S., Ntouyas, S.K., Agarwal, P., Tariboon, J.: Noninstantaneous impulsive inequalities via conformable fractional calculus. J. Inequal. Appl. 2018, 261 (2018)

34. Srivastava, H.M.: Special Functions in Fractional Calculus and Related Fractional Differintegral Equations. World Scientific, Singapore (2014)

35. Srivastava, H.M.: Some families of Mittag-Leffler type functions and associated operators of fractional calculus. J. Pure Appl. Math. 7(2), 123-145 (2016)

36. Srivastava, H.M., Frasin, B.A., Pescar, V.: Univalence of integral operators involving Mittag-Leffler functions. Appl. Math. Inf. Sci. 11, 635-641 (2017)

37. Wiman, A.: Über die Nullstellun der Funktionen $E_{\alpha} x$. Acta Math. 29, 217-234 (1905)

38. Wiman, A.: Uber den fundamental satz in der theorie der funcktionen, $E_{\alpha}$ x. Acta Math. 29, 191-201 (1905)

Submit your manuscript to a SpringerOpen ${ }^{\odot}$ journal and benefit from:

- Convenient online submission

- Rigorous peer review

Open access: articles freely available online

High visibility within the field

- Retaining the copyright to your article

Submit your next manuscript at $\boldsymbol{\nabla}$ springeropen.com 